\title{
MicroRNA-10a/b are regulators of myeloid differentiation and acute myeloid leukemia
}

\author{
LAIXI BI, LAN SUN, ZHENLIN JIN, SHENGHUI ZHANG and ZHIJIAN SHEN \\ Department of Hematology, The First Affiliated Hospital of Wenzhou Medical University, \\ Wenzhou, Zhejiang 325000, P.R. China
}

Received December 16, 2015; Accepted September 7, 2017

DOI: $10.3892 / \mathrm{ol} .2018 .8050$

\begin{abstract}
MicroRNAs (miRs) have been demonstrated to perform important roles in normal hematopoiesis and leukemogenesis. Accumulating evidence suggests that miR-10a and miR-10b may behave as novel oncogenes or tumor suppressors in human cancer. The present study reported the function of the miR-10 family in myeloid differentiation and acute myeloid leukemia (AML). The levels of miR-10a/b expression were increased in AML cases compared with normal controls, particularly in M1, M2 and M3 subtypes. The levels of $\mathrm{miR}-10 \mathrm{a} / \mathrm{b}$ expression were also upregulated in patients with nucleophosmin-mutated AML and AML patients with $\mathrm{t}(8 ; 21)$ and $t(9 ; 11)$, compared with the normal control. In addition, the role of $\mathrm{miR}-10 \mathrm{a} / \mathrm{b}$ in regulating myeloid differentiation and leukemogenesis was investigated. The results indicated that miR-10a/b expression was able to promote the proliferation of human promyelocytic leukemia cells, while suppressing the granulocytic and monocytic differentiation of the leukemia cells. These findings suggested that abnormal high expression of $\mathrm{miR}-10 \mathrm{a} / \mathrm{b}$ may result in unlimited proliferation of immature blood progenitors and repression of mature blood cell differentiation and maturation, thus leading to the occurrence of AML. miR-10a/b may be developed as novel therapeutic targets for the treatment of AML.
\end{abstract}

\section{Introduction}

All mature blood cells are derived from hematopoietic stem cells, and this process is known as hematopoiesis. In a healthy adult, $10^{11}-10^{12}$ new blood cells are produced daily in order to maintain a steady state in the peripheral circulation (1). Granulocytes are a class of white blood cells marked with the

Correspondence to: Dr Zhijian Shen, Department of Hematology, The First Affiliated Hospital of Wenzhou Medical University, 1 XueFu Road, Nanbaixiang, Ouhai, Wenzhou, Zhejiang 325000, P.R. China

E-mail: shenzhijian-wz@126.com

Key words: microRNA-10a/b, acute myeloid leukemia, myeloid differentiation presence of granules in their cytoplasm, which serve important roles in the innate immune system and are involved in chemotaxis, phagocytosis and bactericidal action (2). Monocytes are the largest of all leukocytes, characterized by a granulated cytoplasm. Monocytes also have multiple roles in immune function, including replenishing resident macrophages and differentiating into macrophages and dendritic cells to elicit an immune response $(3,4)$. Granulocytes and monocytes are derived from the common myeloid progenitors. Granulocytic and macrophage-like differentiation and maturation are important components of normal hematopoiesis (5). Blocking myeloid differentiation results in acute myeloid leukemia (AML). AML is characterized by the reduced production of functional blood cells and overproduction and accumulation of immature white blood cells. The symptoms of AML include fatigue, shortness of breath, easy bruising and bleeding and an increased risk of infection (6). AML is the most common type of acute leukemia affecting adults, and the incidence of AML increases with age $(7,8)$. The French-American-British (FAB) classifies AML into eight subtypes (M0-M7) according to cell morphology and maturation (9). From M1 to M5, AML cases involving granulocytic and macrophage-like differentiation blockage account for $85 \%$ of adult AML cases $(9,10)$. Granulocytic and monocytic differentiation is regulated by multiple factors, including key transcriptional factors (PU.1, CCAAT/enhancer-binding protein $\alpha$, interferon regulatory factor 8 , TAL bHLH transcription factor 1 and runt-related transcription factor 1) (11), cytokines [granulocyte colony-stimulating factor (CSF), macrophage-CSF and interleukin-3] (12) and non-coding RNAs (13).

MicroRNAs (miRNA) are a class of small non-coding RNAs, which regulate gene expression post-transcriptionally through degradation of mRNA or inhibition of mRNA translation. miRNAs have been reported to be involved in numerous physiological and pathological biological processes (14). A number of miRNAs have been indicated to be involved in myeloid differentiation and AML progression, including the miR-223, miR-142-3p, miR-29 and miR-181 families (15-17). The miR-10 family consists of two members, miR-10a and miR-10b, which are located at chromosome 17 and 2, respectively (18). Accumulating evidence suggests that miR-10a/b may act as novel oncogenes in various types of human cancer, including metastatic breast cancer, pancreatic cancer, esophageal cancer, hepatocellular carcinoma, nasopharyngeal 
carcinoma and colorectal cancer (19-28). These findings suggest that miR-10a and miR-10b are strongly expressed in highly metastatic cancer and serve important roles in cancer metastasis. miR-10a and miR-10b have also been reported to be upregulated in nucleophosmin (NPM1)-mutated AMLs $(29,30)$. However, the regulatory role of miR-10a/b in AML remains unknown.

In the present study, the expression of miR-10a and miR-10b was examined in a number of AML cases and healthy donor controls. It was revealed that the expression of miR-10a and miR-10b was increased in AML cases compared with healthy controls, particularly in M1, M2 and M3 subtypes. Additionally, the levels of miR-10a and miR-10b were upregulated in AML patients with mutated NPM1, and also AML patients with $\mathrm{t}(8 ; 21)$ and $\mathrm{t}(9 ; 11)$, compared with the normal controls. Furthermore, the roles of miR-10a and miR-10b in regulating granulocytic and monocytic differentiation and AML progression were investigated. The results indicated that miR-10a and miR-10b were able to promote the proliferation of human promyelocytic leukemia HL-60 cells, while suppressing the granulocytic and monocytic differentiation of HL-60 cells. These findings suggested that abnormal high expression of miR-10a and miR-10b may result in unlimited proliferation of immature blood progenitors and the repression of mature blood cell differentiation and maturation, thus leading to the occurrence of AML. Therefore, miR-10a and miR10b may be developed as new therapeutic targets of AML.

\section{Materials and methods}

Human samples. The peripheral blood samples of patients with AML (89 patients) and normal volunteers (65 patients) were obtained from The First Affiliated Hospital of Wenzhou Medical University (Nanbaixiang, Ouhai, Wenzhou, Zhejiang) from January to December 2015. The gender ratio of the patients and controls was approximately 1:1 and aged between 2-60 years old. Written informed consent to perform the biological studies was obtained from all examined patients. The present study was approved by the Ethics Committees of The First Affiliated Hospital of Wenzhou Medical University. Mononuclear cells were isolated from the peripheral blood samples of patients with AML and normal volunteers using lymphocyte separation medium (Lonza Walkersville, Inc., Walkersville, MD, USA). The peripheral blood samples were first diluted with phosphate-buffered saline (PBS; 1:4) and then plated on the lymphocyte separation medium. The middle layer of mononuclear cells was collected following centrifugation at $1,000 \mathrm{x} \mathrm{g}$ for $30 \mathrm{~min}$ at room temperature.

Cell culture and transfections. The human leukemia HL-60 cells (ATCC, Manassas, VA, USA) were grown in Iscove's modified Dulbecco's medium (Invitrogen; Thermo Fisher Scientific, Waltham, MA, USA) supplemented with $10 \%$ fetal bovine serum (Gibco; Thermo Fisher Scientific, Inc.,), $50 \mathrm{U} / \mathrm{ml}$ penicillin and $50 \mu \mathrm{g} / \mathrm{ml}$ streptomycin (Sigma-Aldrich; Merck KGaA, Darmstadt, Germany) at $37^{\circ} \mathrm{C}$ in a $5 \% \mathrm{CO}_{2}$ cell culture incubator. All-trans-retinoic acid (ATRA; $50 \mathrm{ng} / \mathrm{ml}$; Sigma-Aldrich; Merck KGaA) and phorbol myristate acetate (PMA; $2 \mu$ M; Sigma-Aldrich; Merck KGaA) were used to induce HL-60 cells to undergo granulocytic and monocytic differentiation. miR-10a mimics, miR-10b mimics and scramble control were obtained from Dharmacon (GE Healthcare Life Sciences, Little Chalfont, UK) and transfected with DharmFECT1 (Dharmacon; GE Healthcare Life Sciences) into HL-60 cells at a final concentration of $50 \mathrm{nM}$.

May-Grünwald Giemsa staining. HL-60 cells undergoing granulocytic and monocytic differentiation were harvested at indicated time. Cells were smeared on glass slides, fixed in $100 \%$ methanol for $10 \mathrm{~min}$ at room temperature, then stained with May- Grünwald/Giemsa (cat. no. BA-4017; BaSO Diagnostics Inc., Zhuhai, China) for $5 \mathrm{~min}$ at room temperature, and analyzed at $\mathrm{x} 400$ magnification under an inverted microscope (Nikon TE2000; Nikon Corporation, Tokyo, Japan) equipped with a digital camera.

RNA extraction, $C D N A$ synthesis and reverse transcriptionquantitative polymerase chain reaction $(R T-q P C R)$ assays. Total RNA was extracted from cultured cells and mononuclear cells isolated from peripheral blood using TRIzol reagent (Invitrogen; Thermo Fisher Scientific, Inc.) according to the manufacturer's protocol. cDNA was synthesized using high-capacity cDNA reverse transcription Kit (Thermo Fisher Scientific, Inc.) from 1-5 $\mu \mathrm{g}$ of total RNA. For detection of microRNA expression, a stem-loop RT primer was used for the reverse transcription of miR-10a/b. For detection of mRNA expression, oligodT primer was used for the reverse transcription of mRNA. RT-qPCR using SYBR ${ }^{\circledast}$ Green qPCR Master Mix (Takara Bio, Inc., Otsu, Japan) was performed in a Bio-Rad CFX96 real-time PCR System (Bio-Rad Laboratories, Inc., CA, USA) according to the manufacturer's protocols. The PCR conditions were as follows: $95^{\circ} \mathrm{C}$ for $30 \mathrm{sec}$, followed by 40 cycles of $95^{\circ} \mathrm{C}$ for $5 \mathrm{sec}$ and $60^{\circ} \mathrm{C}$ for $34 \mathrm{sec}$. The data were normalized using the endogenous U6 small nuclear RNA and GAPDH. The $2^{-\triangle \Delta C q}$ method (31) was used in the analysis of PCR data. Primer sequences are presented in Table I.

Flow cytometry and fluorescence-activated cell sorting analysis (FACS). HL-60 cells were harvested at $48 \mathrm{~h}$ after transfection and were washed twice at $4^{\circ} \mathrm{C}$ in PBS containing $0.5 \%$ fetal bovine serum (Gibco; Thermo Fisher Scientific, Inc.). ATRA- or PMA-induced HL-60 cells were incubated with phycoerythrin-conjugated cluster of differentiation molecule 11B (CD11b) antibody (CD11b04-4; eBioscience; Thermo Fisher Scientific, Inc.) or fluorescein isothiocyanate-conjugated anti-cluster of differentiation CD14 antibody (11-0141-82; eBioscience; Thermo Fisher Scientific, Inc.) at 1:100 dilution for $30 \mathrm{~min}$ at room temperature. Flow cytometry was performed using a C6 flow cytometer instrument (BD Biosciences, Franklin Lakes, NJ, USA) and subsequently analyzed with CFlow Sampler Analysis 1.0.208.2 (BD Biosciences).

Cell proliferation assay. To measure the effect of miRNA mimics on proliferation of HL-60 cells, the cells were incubated in $10 \%$ cell counting kit- 8 solution (Dojindo Molecular Technologies, Inc., Kumamoto, Japan) and diluted in normal culture medium at $37^{\circ} \mathrm{C}$ until color change has taken place. Proliferation rates were determined on day 1, 2, 3 and 4 post-transfection, and quantification was performed on a microtiter plate reader (Spectra Rainbow, Tecan Trading 
Table I. Sequences of primers used in reverse transcriptionquantitative polymerase chain reaction.

\begin{tabular}{|c|c|}
\hline Primer & Sequence $\left(5^{\prime}-3^{\prime}\right)$ \\
\hline miR-10a-RT & $\begin{array}{l}\text { GTCGTATCCAGTGCAGGGTCCGAGG } \\
\text { TATTCGCACTGGATACGACCACAAAT }\end{array}$ \\
\hline miR-10b-RT & $\begin{array}{l}\text { GTCGTATCCAGTGCAGGGTCCGAGG } \\
\text { TATTCGCACTGGATACGCACAAATT }\end{array}$ \\
\hline miR-10a-forward & GCGC TACCCTGTAGATCCG \\
\hline miR-10a-reverse & GTGCTACCCTGTAGAAC \\
\hline miR-10b-forward & AGCTGTTCAGTGCACTACAGA \\
\hline miR-10b-reverse & GTGCTACCCTGTAGAAC \\
\hline miR-10a-probe & FAM-CCTGTAGATCCGAATTTG-MGB \\
\hline miR-10b-probe & FAM-CCTGTAGAACCGAATTTG-MGB \\
\hline U6-RT & AAAATATGGAACGCTTCACGAATTTG \\
\hline U6-forward & CTCGCTTCGGCAGCACATATACT \\
\hline U6-reverse & ACGCTTCACGAATTTGCGTGTC \\
\hline U6-probe & $\begin{array}{l}\text { FAM-CCATGCTAATCTTCTCTGTA- } \\
\text { MGB }\end{array}$ \\
\hline CD14-forward & GACCTAAAGATAACCGGCACC \\
\hline CD14-reverse & GCAATGCTCAGTACCTTGAGG \\
\hline CD11b-forward & CAGACAGGAAGTAGCAGCTCCT \\
\hline CD11b-reverse & CTGGTCATGTTGATGAAGGTGCT \\
\hline GAPDH-forward & TCAACGACCACTTTGTCAAGCTCA \\
\hline GAPDH-reverse & GCTGGTGGTCCAGGGGTCTTACT \\
\hline CSF1R-forward & GGGAATCCCAGTGATAGAGCC \\
\hline CSF1R-reverse & TTGGAAGGTAGCGTTGTTGGT \\
\hline CSF3R-forward & TCAAGTTGGTGCTATGGCAAGG \\
\hline CSF3R-reverse & GCTCCCAGTCTCCACAGAATC \\
\hline
\end{tabular}

$\mathrm{CD}$, cluster of differentiation; CSF1R, colony-stimulating factor 1 receptor; CSF3R, colony stimulating factor 3 receptor; MGB, minor groove binder; FAM, fluorescein; RT, reverse transcription.

AG, Männedorf, Switzerland) at OD450 $\mathrm{nm}$ according to the manufacturer's protocol.

Statistical analysis. Comparisons between multiple groups were analyzed using one-way analysis of variance and the least significant difference post hoc test using GraphPad Prism 5 v5.01 (Graph Pad Software, Inc., La Jolla, CA, USA). Comparisons between two groups were evaluated by independent sample t-test (two-tailed). $\mathrm{P} \leq 0.05$ was considered to indicate a statistically significant difference. Data are presented as the mean \pm standard deviation.

\section{Results}

miR-10a and miR-10b are significantly increased in patients with $A M L$. To accurately examine the expression of miR-10a and miR-10b in AML, TaqMan quantitative PCR was used to detect the levels of miR-10a and miR-10b in peripheral blood mononuclear cells (PBMNCs) derived from 89 patients with AML and 65 healthy donors (Fig. 1).

Compared with the normal controls, the expression of miR-10a increased significantly in AML cases $(\mathrm{P}<0.001$; Fig. 1A). These AML cases that were analyzed included major FAB subtypes from M1 to M5, including 13 cases of M1, 28 cases of M2, 21 cases of M3, 10 cases of M4 and 17 cases of M5. The expression level of miR-10a was also compared in the different AML subtypes, and it was indicated that the expression of miR-10a in M1, M2 and M3 AML subtypes was increased compared with M4, M5 and normal controls. The expression of miR-10a in M4 and M5 subtypes was also higher compared with normal controls, but this difference was not statistically significant (Fig. 1B).

In addition, the expression of miR-10a and miR-10b based on cytogenetic karyotypes was also compared in 57 patients with AML, whose cytogenetic karyotype and gene mutation had been detected. The results showed that miR-10a was significantly upregulated in AML patients with $t(8 ; 21)$ and $t(9 ; 11)$, but not in patients with AML with $\mathrm{t}(15 ; 17)$ and $\mathrm{t}(10 ; 16)$ when compared with the normal controls (Fig. 1C). In addition, the expression level of miR-10a was also upregulated in patients with NPM1-mutated AML compared with normal controls, and patients with AML and wild-type NPM1 (Fig. 1D), which was consistent with previous studies $(29,30,32)$.

Similar to miR-10a expression, the expression of miR-10b also increased significantly in AML cases compared with the normal controls $(\mathrm{P}<0.001$; Fig. 1E). The expression of miR-10b in M1, M2 and M3 AML subtypes was higher compared with the expression in M4, M5 and normal controls (Fig. 1F). Additionally, the expression in M4 and M5 AML subtypes was also a little but not significant higher compared with the expression in normal controls (Fig. 1F). The expression level of miR-10b expression was increased in patients with AML with $t(8 ; 21)$ and $t(9 ; 11)$ compared with the expression in normal controls (Fig. 1G). Additionally, the level of miR-10b was significantly upregulated in patients with NPM1-mutated AML compared with normal controls, and patients with AML and wild-type NPM1 (Fig. 1H). These results indicated the involvement of miR-10a and miR-10b in leukemogenesis.

miR-10a and miR-10b are gradually decreased during granulocytic and monocytic differentiation of human leukemia cells. To examine the possible role of miR-10a and miR-10b in granulocytic and monocytic differentiation and leukemogenesis, the expression level of the two miRNAs was detected during granulocytic and monocytic differentiation of human leukemia HL-60 cells. HL-60 was induced to undergo granulocytic or monocytic differentiation using ATRA or PMA, respectively, as previously reported (33). As Giemsa staining shown, more mature granulocytic cells show polylobular nuclei, more mature monocytes show bluish-gray cytoplasm and a saddle-shaped nucleus (Fig. 2A and B) qPCR was used to detect the level of miR-10a and miR-10b expression at 12, 24, 48 and $72 \mathrm{~h}$ post-induction. The results showed that miR-10a/b decreased significantly during ATRA-induced granulocytic differentiation (Fig. 2C) and PMA-induced monocytic differentiation (Fig. 2D). These findings suggested that miR-10a and miR-10b may perform critical roles in granulocytic/monocytic differentiation. 
A

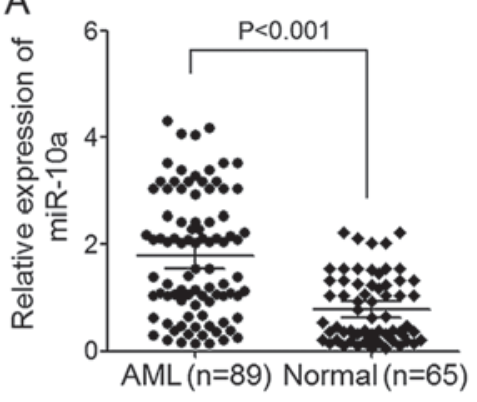

B
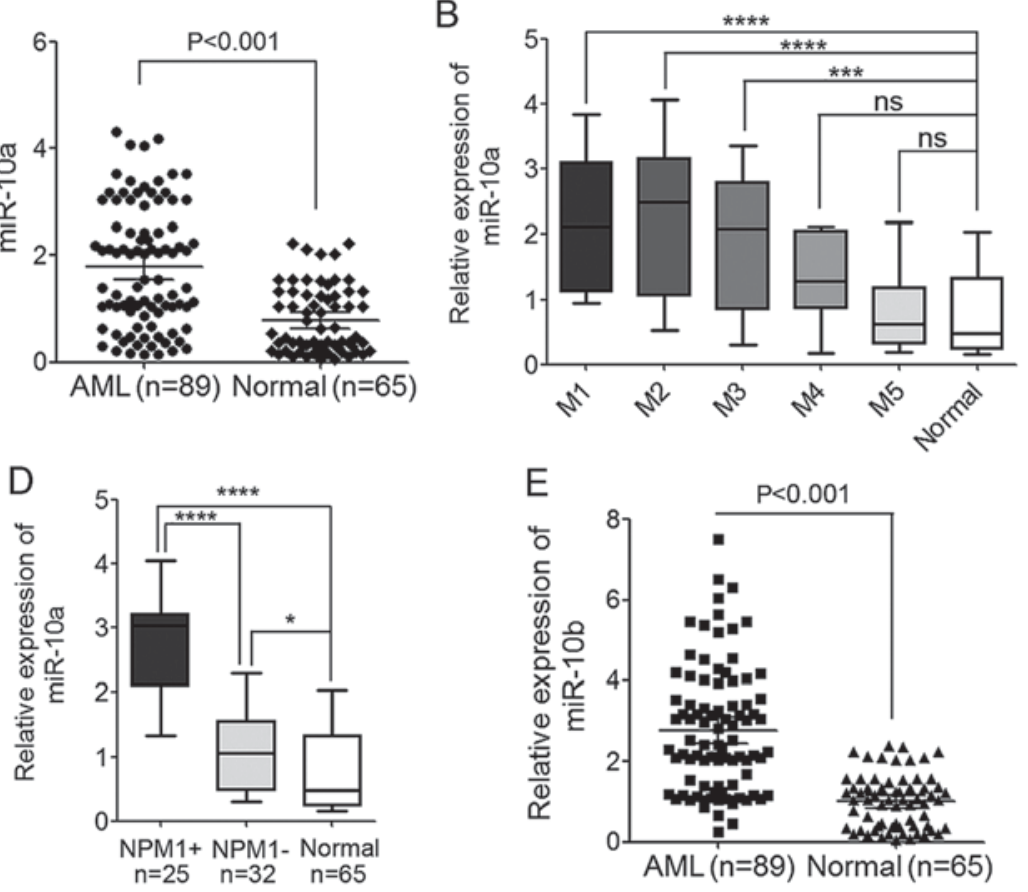

E

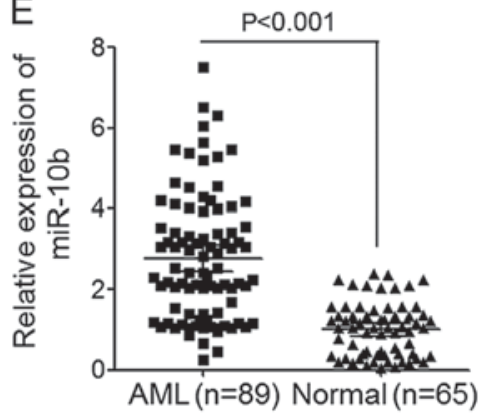

C

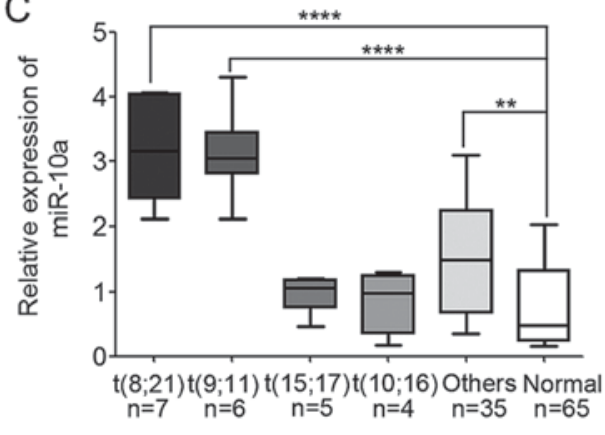

$\mathrm{F}$

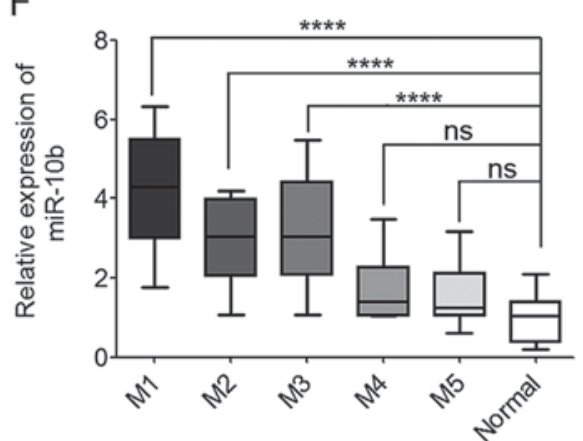

G

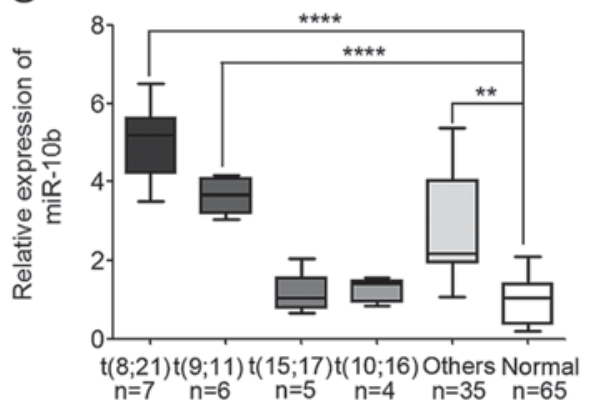

$\mathrm{H}$

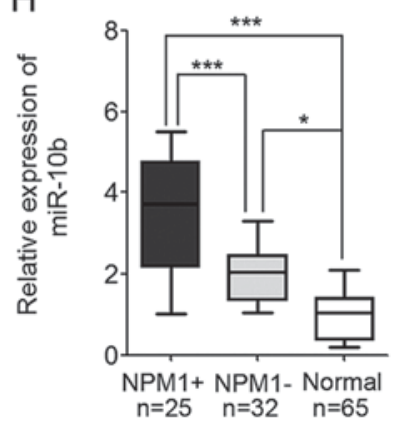

Figure 1. Levels of miR-10a and miR-10b expression are significantly increased in AML cases. (A) The expression level of miR-10a in PBMNCs derived from 89 patients with AML and 65 healthy donors detected by TaqMan quantitative polymerase chain reaction. (B) The expression level of miR-10a in different AML subtypes, from M1 to M5. (C) The expression level of miR-10a in different WHO subtypes of AML patients compared with the normal controls. (D) The expression level of miR-10a in AML patients with mutated and wild-type NPM1 compared with the normal controls. (E) The expression level of miR-10b in PBMNCs derived from 89 AML patients and 65 healthy donors. (F) The expression level of miR-10b in different AML subtypes, from M1 to M5. (G) The expression level of miR-10b in different WHO subtypes of AML patients compared with the normal controls. (H) The expression level of miR-10b in AML patients with mutated and wild-type NPM1 compared with the normal controls. ${ }^{*} \mathrm{P}<0.05,{ }^{* * *} \mathrm{P}<0.01,{ }^{* * *} \mathrm{P}<0.001,{ }^{* * * * *} \mathrm{P}<0.0001$; ns, not significant. AML, acute myeloid leukemia; miR, microRNA; NPM1, nucleophosmin; PBMNC, peripheral blood mononuclear cell; WHO, World Health Organization; NPM1+, NPM1 mutated; NPM1-, wild-type NPM1.

miR-10a promotes proliferation of human leukemia cells and inhibits differentiation of human leukemia cells to granulocytes and monocytes. Given the decrease in miR-10a and miR-10b expression during granulocytic/monocytic differentiation of HL-60 cells, it was questioned whether miR-10a and miR-10b regulate granulocytic/monocytic differentiation or proliferation of leukemia cells. miR-10a mimics were transfected into HL-60 cells. Cell growth rate and differentiation were then examined. miR-10a was successfully overexpressed in HL-60 cells as confirmed by qPCR (Fig. 3A). Cell proliferation rates were determined at days 1,2, 3 and 4 post-transfection, and overexpression of miR-10a in HL-60 cells was observed to be able to significantly promote the proliferation of leukemia cells compared with the scramble control (Fig. 3B).

In addition, the effect of miR-10a on the ability of leukemia cells to differentiate into granulocyte and monocytes was investigated. Transfected HL-60 cells were induced to undergo granulocytic/monocytic differentiation, and the expression of CD11b and CSF3 receptor (CSF3R) were used to estimate the progression of granulocytic differentiation, while the expression of CD14 and CSF1 receptor (CSF1R) were used to estimate the progression of monocytic differentiation. FACS analysis of CD11b and CD14 was also used to examine the granulocytic/monocytic differentiation of HL-60 cells. The results demonstrated that ectopic expression of miR-10a was able to suppress the upregulation of CD11b and CSF3R during ATRA-induced granulocytic differentiation (Fig. 3C) and also was able to decrease the percentage of CD11b-positive cells (Fig. 3D). Ectopic expression of miR-10a also significantly inhibited the upregulation of CD14 and CSF1R during PMA-induced monocytic differentiation (Fig. 3E) and decreased the percentage of CD14-positive cells 
A

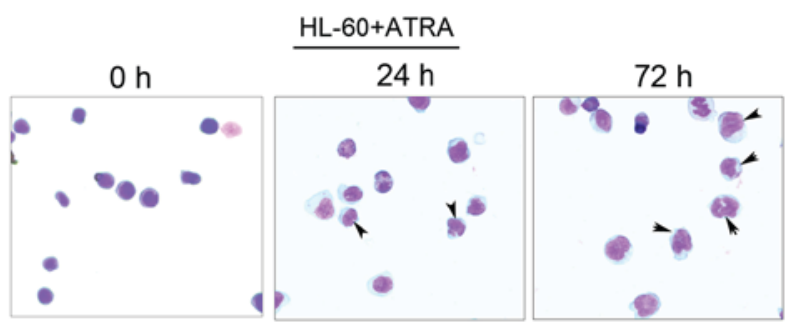

C

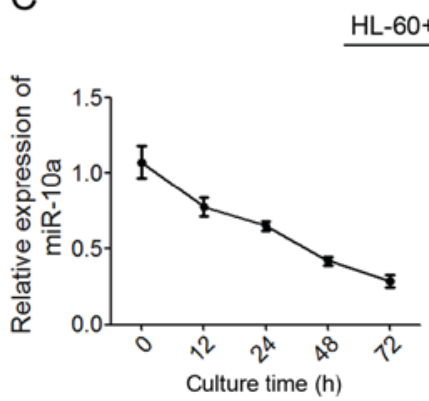

HL-60+ATRA

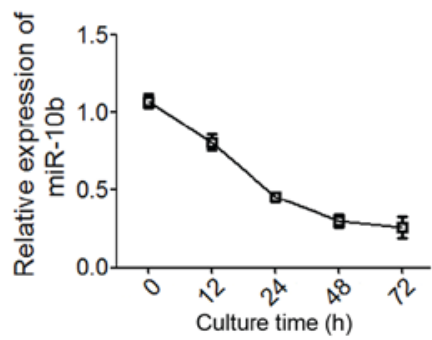

$B$

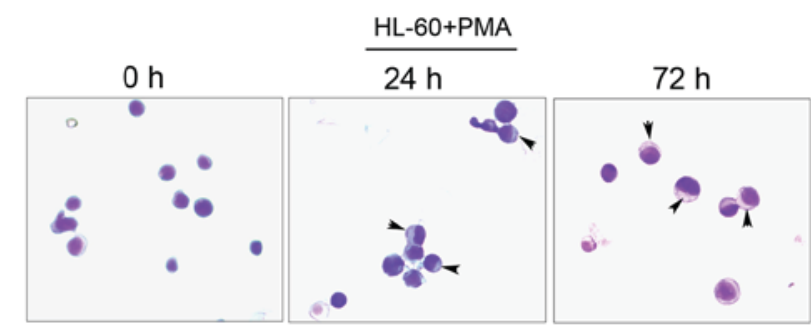

D

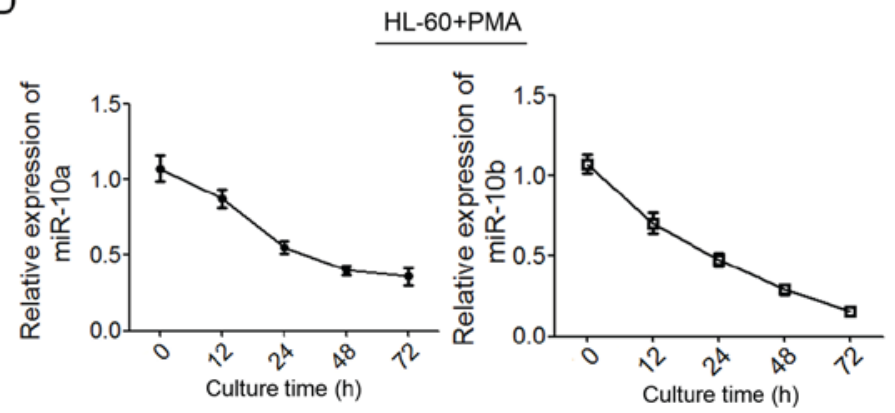

Figure 2. Decreased miR-10a/b expression during granulocytic and monocytic differentiation of HL-60 cells. (A and B) May-Grünwald Giemsa staining of HL-60 cells that were induced by ATRA or PMA for 0, 24 and $72 \mathrm{~h}$, respectively. The differentiated macrophages and monocytes were indicated with arrows. (C) Changes in expression level of miR-10a and miR-10b during ATRA-induced HL-60 granulocytic differentiation at 12, 24, 48 and $72 \mathrm{~h}$ post-induction. (D) Changes in the level of (C) miR-10a and (D) miR-10b expression during PMA-induced monocytic differentiation. Data are presented as the mean \pm standard deviation. miR, microRNA; ATRA, all-trans-retinoic acid; PMA, phorbol myristate acetate.

(Fig. 3F), indicating that miR-10a performs a negative role in granulocytic/monocytic differentiation of HL-60 cells.

miR-10b promotes cell proliferation and suppresses granulocytic and monocytic differentiation. The role of miR-10b in regulating granulocytic/monocytic differentiation and cell proliferation of leukemia cells was also detected, in the same way as miR-10a. miR-10b was successfully overexpressed in HL-60 cells as confirmed by qPCR (Fig. 4A). Cell proliferation rates were determined at days 1, 2, 3 and 4 post-transfection, and ectopic expression of miR-10b was observed to promote the proliferation of leukemia cells (Fig. 4B). In addition, it was revealed that overexpression of miR-10b suppressed the upregulation of CD11b and CSF3R during ATRA-induced granulocytic differentiation (Fig. 4C) and also decreased the percentage of CD11b-positive cells (Fig. 4D). Furthermore, the overexpression of miR-10b also significantly inhibited the upregulation of CD14 and CSF1R during PMA-induced monocytic differentiation (Fig. 4E) and decreased the percentage of CD14-positive cells (Fig. 4F), indicating that miR-10b has a negative role in granulocytic/monocytic differentiation of HL-60 cells.

\section{Discussion}

Several studies have investigated the association between abnormalmiR-10a/bexpression and the risk of developing various types of cancer, but the results are inconsistent $(19,21,23,26)$. Accumulating evidence suggests that miR-10a and miR-10b may behave as novel oncogenes in human cancer $(26-28,34,35)$. The detection of upregulation of miR-10a expression may serve as potential biomarkers of aggressive progression and poor prognosis in cervical cancer (34) miR-10b has also been reported to promote cell invasion by targeting homeobox (HOX) D10 in gastric cancer (35). However, debate exists on whether $\mathrm{miR}-10 \mathrm{a} / \mathrm{b}$ acts as a tumor suppressor or oncogene in human cancer (34-37). Kim et al (36) reported that miR-10b may act as a tumor suppressive gene in gastric carcinogenesis. The loss of miR-10a was also reported to activate lactoperoxidase to induce intestinal neoplasia through cooperating with activated Wnt signaling in female mice (37). However, the aberrant expression and the potential role of $\mathrm{miR}-10 \mathrm{a} / \mathrm{b}$ in AML are largely unknown, with the exception that miR-10a was reported to be expressed at high levels in NPM1-mutated AML (38) and that the level of serum miR-10a was also investigated as a prognostic biomarker for AML (39).

In the present study, the expression of miR-10a and miR-10b was examined in a number of patients with AML and healthy controls. Additionally, the potential application of the levels of miR-10a and miR-10b expression in AML diagnosis was investigated. The results indicated that miR-10a and miR-10b were significantly upregulated in AML samples. The upregulation of miR-10a and miR-10b in AML indicated that $\mathrm{miR}-10 \mathrm{a} / \mathrm{b}$ may serve as potential biomarkers for diagnosis of AML. Several innate properties of miRNAs make them attractive as potential biomarkers. miRNAs are small and stable against degradation and can be detected easily by specific and sensitive RT-qPCR in small amount samples. In addition, miRNAs are also detectable in bodily fluids, including serum, plasma, saliva, urine and tears $(40,41)$. Furthermore, expression profiles of miRNAs in the plasma and/or serum of cancer patients may reflect the change in miRNA expression in tumor cells (42). Circulating miRNAs may be a novel class of non-invasive biomarkers for cancer diagnostic and prognostic information $(43,44)$. The differential expression of miR-10a/b in different AML 


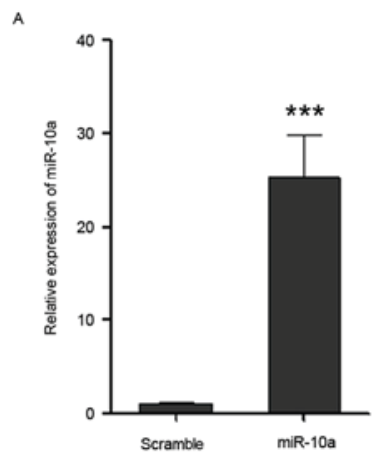

$\circ$
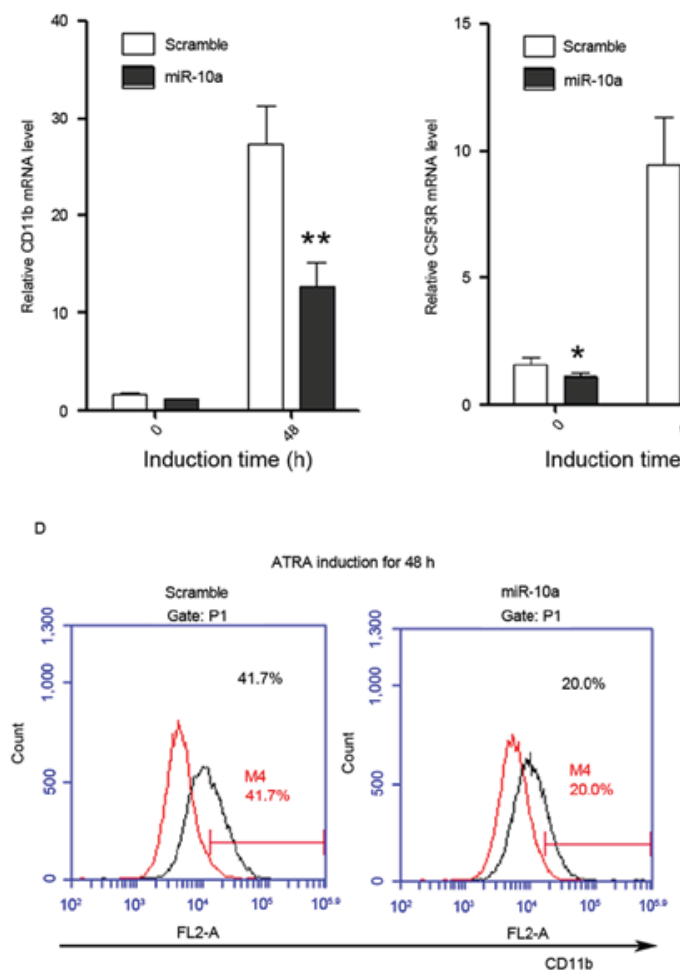

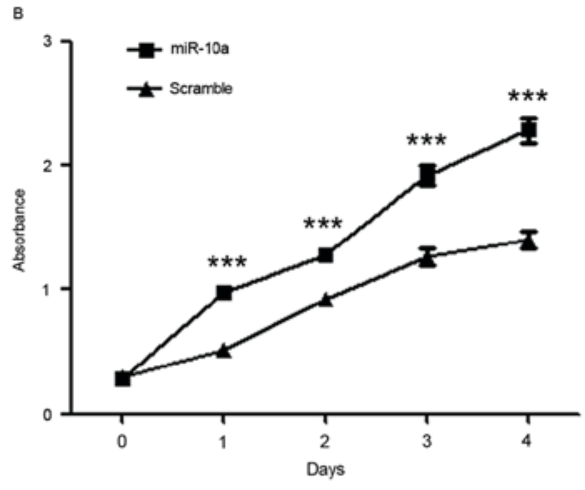

E

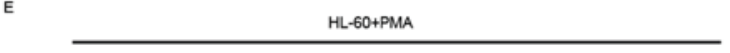

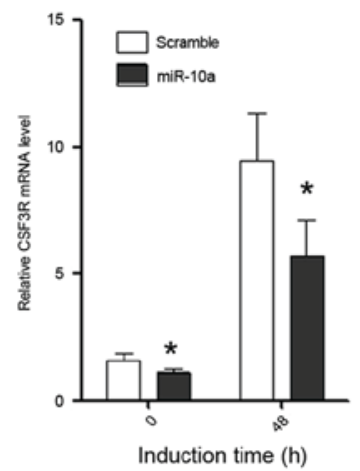
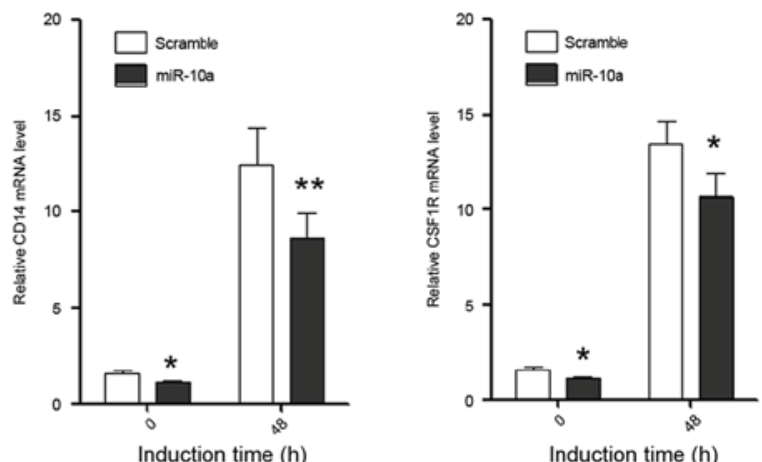

Induction time (h)
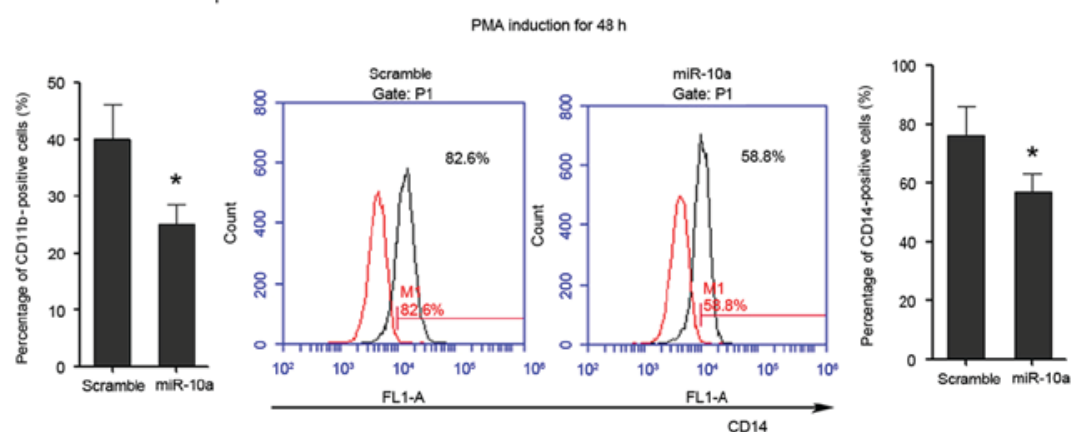

Figure 3. miR-10a promotes the proliferation of HL-60 cells, while inhibits the differentiation of HL-60 cells to granulocytes and monocytes. (A) miR-10a is overexpressed in HL-60 cells as confirmed by quantitative polymerase chain reaction. (B) The growth of HL-60 cells at day 0, 1, 2,3 and 4 post-transfection, which was detected by Cell Counting Kit-8 assay. (C) The expression level of granulocytic marker CD11b and CSF3R in untreated and ATRA-treated (duration, $48 \mathrm{~h}$ ) cells that overexpress miR-10a. (D) CD11b FACS analysis showed that overexpression of miR-10a was able to delay ATRA-induced granulocytic differentiation of HL-60 cells. The percentage of CD11b-positive cells was calculated using the black traces referring to cells stained with CD11b antibody against the red traces referring to the same cells not stained with CD11b antibody. (E) The relative expression of monocytic marker CD14 and CSF1R in untreated and PMA-treated (duration, $48 \mathrm{~h}$ ) cells that overexpress miR-10a. (F) CD14 FACS analysis showed that overexpression of miR-10a delayed PMA-induced monocytic differentiation of HL-60 cells. The percentage of CD14-positive cells was calculated in the same way. Data are presented as the mean \pm standard deviation $(\mathrm{n}=3)$. All the comparisons are made between scramble and miR-10a groups. ${ }^{*} \mathrm{P}<0.05 ;{ }^{* *} \mathrm{P}<0.01 ;{ }^{* * *} \mathrm{P}<0.001$. miR, microRNA; CSF3R, colony-stimulating factor 3 receptor; ATRA, all-trans-retinoic acid; PMA, phorbol myristate acetate; CD, cluster of differentiation; CSF1R, colony-stimulating factor 1 receptor; FACS, fluorescence-activated cell sorting.

subtypes should be validated in more clinical samples to develop a novel method for subtyping AML according to miRNA expression.

In addition, to the best of our knowledge, there has not been any study that investigated the role of miR-10a and miR-10b in the progression of AML. The present study revealed that the exogenous expression of miR-10a and miR-10b in HL-60 cells decreased the maturation of HL-60 cells to granulocytes and monocytes, as well as the expression of the granulocytic and monocytic differentiation markers. The overexpression of
miR-10a and miR-10b was able to promote the proliferation of leukemia cells. It was hypothesized that the abnormal upregulation of miR-10a/b in blood progenitor cells would result in the overproduction and accumulation of immature white blood cells, as well as the inhibition of granulocyte and monocyte maturation, which results in leukemogenesis. The oncogenic role of $\mathrm{miR}-10 \mathrm{a} / \mathrm{b}$ in the progression of AML will be investigated in animal models.

In humans, miR-10 is co-expressed with a set of Hox genes and has been demonstrated to regulate the translation 

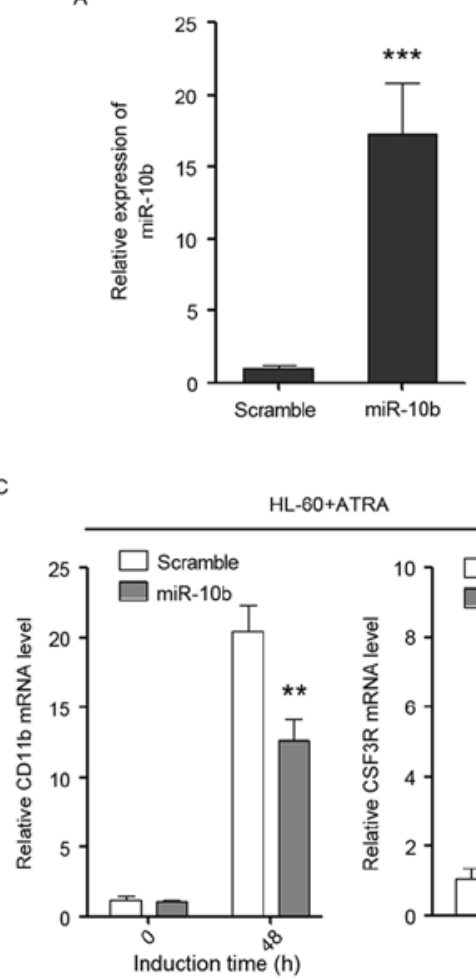

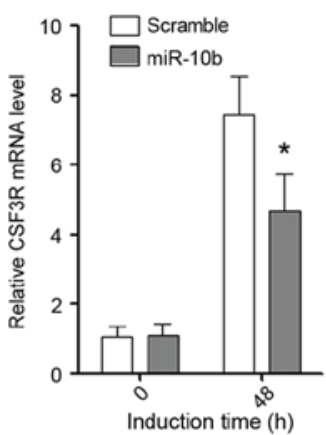

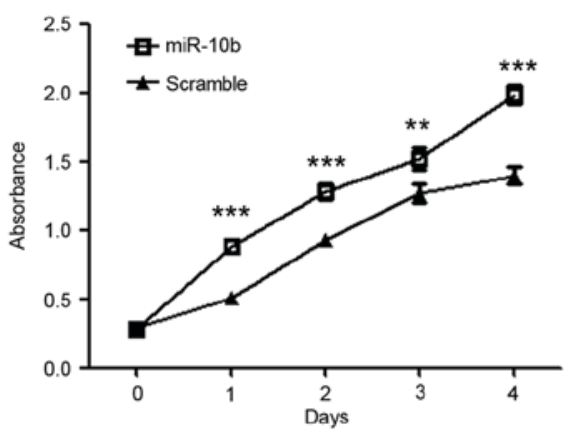

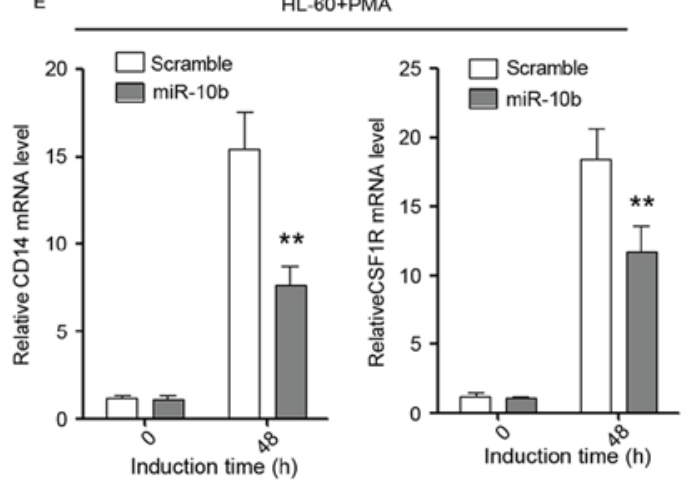

D

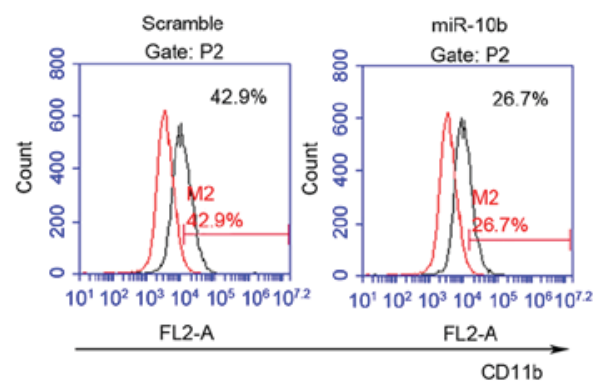

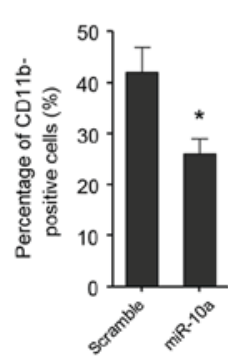

PMA induction for $48 \mathrm{~h}$

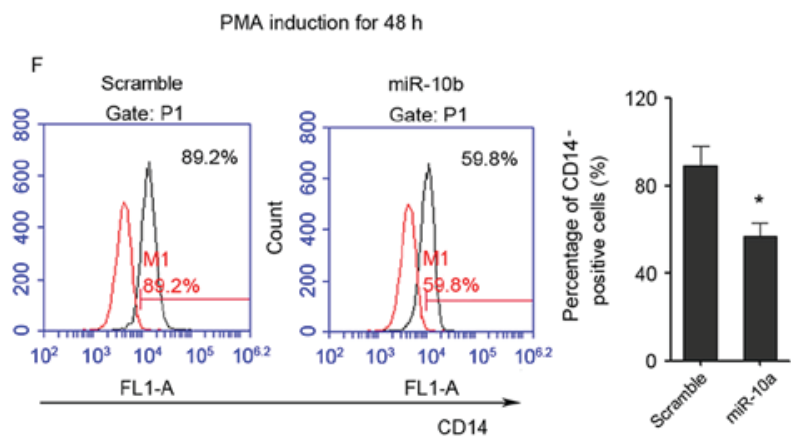

Figure 4. miR-10b promotes the proliferation of HL-60 cells, while inhibits the differentiation of HL-60 cells to granulocytes and monocytes. (A) The overexpression of miR-10b in HL-60 cells as confirmed by quantitative polymerase chain reaction. (B) The growth of HL-60 cells at day $0,1,2,3$ and 4 post-transfection as detected by Cell Counting Kit- 8 assay. (C) The expression level of granulocytic marker CD11b and CSF3R in untreated and ATRA-treated (duration, $48 \mathrm{~h}$ ) cells that overexpress miR-10b. (D) CD11b FACS analysis showed that enforced expression of miR-10b delayed ATRA-induced granulocytic differentiation of HL-60 cells. (E) The relative expression of monocytic marker CD14 and CSF1R in untreated and PMA-treated (duration, 48 h) cells that overexpress miR-10b. (F) CD14 FACS analysis showed that enforced expression of miR-10b delayed PMA-induced monocytic differentiation of HL-60 cells. Data are presented as the mean \pm standard deviation $(n=3) ;{ }^{*} \mathrm{P}<0.05 ;{ }^{* *} \mathrm{P}<0.01 ;{ }^{* * * *} \mathrm{P}<0.001$. All the comparisons are made between scramble and miR-10a groups. miR, microRNA; CSF3R, colony-stimulating factor 3 receptor; CD, cluster of differentiation; CSF1R, colony-stimulating factor 1 receptor; ATRA, al-trans-retinoic acid; PMA, phorbol myristate acetate; FACS, fluorescence-activated cell sorting.

of Hox transcripts $(45,46)$. Hox genes perform crucial roles during development and are also involved in the tumorigenesis of various types of cancer (46). HOXA1 has been identified as a direct target of miR-10a in gastric cancer, megakaryocytopoiesis and pancreatic cancer (47). The miR-10 family has also been reported to facilitate cancer by regulating ribosome biogenesis and consequently global protein production (48). Therefore, it was hypothesized that miR-10a and miR-10b modulate granulocytic/monocytic differentiation and AML carcinogenesis through regulating Hox gene expression or ribosome biogenesis. The possible mechanism by which miR-10a and miR-10b regulate granulocytic and monocytic differentiation and AML carcinogenesis requires additional investigation.

\section{Acknowledgements}

Not applicable.

\section{Funding}

The present study was supported by grants from the National Natural Science Foundation of China (grant nos. 81100355 and 81172613).

\section{Availability of data and materials}

All data generated or analyzed during the present study are included in this published article. 


\section{Authors' contributions}

ZS and LB conceived and designed the study. LB performed the majority of experiments. LS and ZJ collected the clinical samples. SZ helped to perform the cell culture experiments. LB and ZS wrote the manuscript. All authors have read and approved this manuscript.

\section{Ethics approval and consent to participate}

The present study was approved by the Ethics Committees of The First Affiliated Hospital of Wenzhou Medical University and written informed consent was obtained from all patients.

\section{Consent for publication}

Written informed consent was obtained from all examined patients for the publication of their data.

\section{Competing interests}

The authors declare that they have no competing interests.

\section{References}

1. Birbrair A and Frenette PS: Niche heterogeneity in the bone marrow. Ann N Y Acad Sci 1370: 82-96, 2016.

2. Morrison SJ and Kimble J: Asymmetric and symmetric stem-cell divisions in development and cancer. Nature 441: 1068-1074, 2006

3. Falini B, Tiacci E, Martelli MP, Ascani S and Pileri SA: New classification of acute myeloid leukemia and precursor-related neoplasms: Changes and unsolved issues. Discov Med 10: 281-292, 2010

4. Jemal A, Thomas A, Murray $\mathrm{T}$ and Thun M: Cancer statistics, 2002. CA Cancer J Clin 52: 23-47, 2002.

5. Döhner H, Weisdorf DJ and Bloomfield CD: Acute myeloid leukemia. N Engl J Med 373: 1136-1152, 2015.

6. GBD 2015 Disease and Injury Incidence and Prevalence Collaborators: Global, regional, and national incidence, prevalence, and years lived with disability for 310 diseases and injuries, 1990-2015: A systematic analysis for the global burden of disease study 2015. Lancet 388: 1545-1602, 2016.

7. Das A, Sinha M, Datta S, Abas M, Chaffee S, Sen CK and Roy S: Monocyte and macrophage plasticity in tissue repair and regeneration. Am J Pathol 185: 2596-2606, 2015.

8. Rua R and McGavern DB: Elucidation of monocyte/macrophage dynamics and function by intravital imaging. J Leukoc Biol 98 319-332, 2015.

9. Bennett JM, Catovsky D, Daniel MT, Flandrin G, Galton DA, Gralnick HR and Sultan C: Proposals for the classification of the acute leukaemias. French-american-british (FAB) co-operative group. Br J Haematol 33: 451-458, 1976.

10. Garzon R, Volinia S, Liu CG, Fernandez-Cymering C, Palumbo T, Pichiorri F, Fabbri M, Coombes K, Alder H, Nakamura T, et al: MicroRNA signatures associated with cytogenetics and prognosis in acute myeloid leukemia. Blood 111: 3183-3189, 2008.

11. Rosenbauer F and Tenen DG: Transcription factors in myeloid development: Balancing differentiation with transformation. Nat Rev Immunol 7: 105-117, 2007.

12. Egeland T, Steen R, Quarsten H, Gaudernack G, Yang YC and Thorsby E: Myeloid differentiation of purified CD34+ cells after stimulation with recombinant human granulocyte-monocyte colony-stimulating factor (CSF), granulocyte-CSF, monocyteCSF, and interleukin-3. Blood 78: 3192-3199, 1991.

13. Chen CZ, Li L, Lodish HF and Bartel DP: MicroRNAs modulate hematopoietic lineage differentiation. Science 303: 83-86, 2004.

14. Mohr AM and Mott JL: Overview of microRNA biology. Semin Liver Dis 35: 3-11, 2015.
15. Pulikkan JA, Dengler V, Peramangalam PS, Peer Zada AA, Müller-Tidow C, Bohlander SK, Tenen DG and Behre G: Cell-cycle regulator E2F1 and microRNA-223 comprise an autoregulatory negative feedback loop in acute myeloid leukemia. Blood 115: 1768-1778, 2010.

16. Wang XS, Gong JN, Yu J, Wang F, Zhang XH, Yin XL, Tan ZQ, Luo ZM, Yang GH, Shen C and Zhang JW: MicroRNA-29A and microRNA-142-3p are regulators of myeloid differentiation and acute myeloid leukemia. Blood 119: 4992-5004, 2012.

17. Su R, Lin HS, Zhang XH, Yin XL, Ning HM, Liu B, Zhai PF, Gong JN, Shen C, Song L, et al: miR-181 family: Regulators of myeloid differentiation and acute myeloid leukemia as well as potential therapeutic targets. Oncogene 34: 3226-3239, 2015.

18. Havelange V, Ranganathan P, Geyer S, Nicolet D, Huang X, Yu X, Volinia S, Kornblau SM, Andreeff M, Croce CM, et al: Implications of the miR-10 family in chemotherapy response of NPM1-mutated AML. Blood 123: 2412-5, 2014.

19. Ma L, Teruya-Feldstein J and Weinberg RA: Tumour invasion and metastasis initiated by microRNA-10b in breast cancer. Nature 449: 682-688, 2007.

20. Ma L: Role of miR-10b in breast cancer metastasis. Breast Cancer Res 12: 210, 2010.

21. Sasayama T, Nishihara M, Kondoh T, Hosoda K and Kohmura E: MicroRNA-10b is overexpressed in malignant glioma and associated with tumor invasive factors, uPAR and RhoC. Int J Cancer 125: 1407-1413, 2009.

22. Teplyuk NM, Mollenhauer B, Gabriely G, Giese A, Kim E, Smolsky M, Kim RY, Saria MG, Pastorino S, Kesari S and Krichevsky AM: MicroRNAs in cerebrospinal fluid identify glioblastoma and metastatic brain cancers and reflect disease activity. Neuro-Oncol 14: 689-700, 2012.

23. Chai G, Liu N, Ma J, Li H, Oblinger JL, Prahalad AK, Gong M, Chang LS, Wallace M, Muir D, et al: MicroRNA-10b regulates tumorigenesis in neurofibromatosis type 1. Cancer Sci 101: 1997-2004, 2010.

24. Tian Y, Luo A, Cai Y, Su Q, Ding F, Chen H and Liu Z: MicroRNA-10b promotes migration and invasion through KLF4 in human esophageal cancer cell lines. J Biol Chem 285: 7986-7994, 2010.

25. Nakata K, Ohuchida K, Mizumoto K, Kayashima T, Ikenaga N, Sakai H, Lin C, Fujita H, Otsuka T, Aishima S, et al: MicroRNA-10b is overexpressed in pancreatic cancer, promotes its invasiveness, and correlates with a poor prognosis. Surgery 150: 916-922, 2011.

26. Li G, Wu Z, Peng Y, Liu X, Lu J, Wang L, Pan Q, He ML and Li XP: MicroRNA-10b induced by epstein-barr virus-encoded latent membrane protein-1 promotes the metastasis of human nasopharyngeal carcinoma cells. Cancer Lett 299: 29-36, 2010.

27. Ladeiro Y, Couchy G, Balabaud C, Bioulac-Sage P, Pelletier L, Rebouissou S and Zucman-Rossi J: MicroRNA profiling in hepatocellular tumors is associated with clinical features and oncogene/tumor suppressor gene mutations. Hepatology 47: 1955-1963, 2008

28. Yamamoto H, Adachi Y, Taniguchi H, Kunimoto H, Nosho K, Suzuki $\mathrm{H}$ and Shinomura Y: Interrelationship between microsatellite instability and microRNA in gastrointestinal cancer. World J Gastroenterol 18: 2745-2755, 2012

29. Jongen-Lavrencic M, Sun SM, Dijkstra MK, Valk PJ and Löwenberg B: MicroRNA expression profiling in relation to the genetic heterogeneity of acute myeloid leukemia. Blood 111: 5078-5085, 2008

30. Garzon R, Garofalo M, Martelli MP, Briesewitz R, Wang L, Fernandez-Cymering C, Volinia S, Liu CG, Schnittger S, Haferlach T, et al: Distinctive microRNA signature of acute myeloid leukemia bearing cytoplasmic mutated nucleophosmin. Proc Natl Acad Sci U S A 105: 3945-3950, 2008.

31. Livak KJ and Schmittgen TD: Analysis of relative gene expression data using real-time quantitative PCR and the 2(-Delta Delta C(T)) Method. Methods 25: 402-408, 2001.

32. Bryant A, Palma CA, Jayaswal V, Yang YW, Lutherborrow M and Ma DD: miR-10a is aberrantly overexpressed in nucleophosmin1 mutated acute myeloid leukaemia and its suppression induces cell death. Mol Cancer 11: 8, 2012.

33. Capitani S, Marchisio M, Neri LM, Brugnoli F, Gonelli A and Bertagnolo V: Phosphoinositide 3-kinase is associated to the nucleus of HL-60 cells and is involved in their ATRA-induced granulocytic differentiation. Eur J Histochem 44: 61-65, 2000 . 
34. Safari A, Seifoleslami M, Yahaghi E, Sedaghati F and Khameneie MK: Retracted article: Upregulation of miR-20a and miR-10a expression levels act as potential biomarkers of aggressive progression and poor prognosis in cervical cancer. Tumour Biol, 2015.

35. Liu Z, Zhu J, Cao H, Ren H and Fang X: miR-10b promotes cell invasion through RhoC-AKT signaling pathway by targeting HOXD10 in gastric cancer. Int J Oncol 40: 1553-1560, 2012.

36. Kim K, Lee HC, Park JL, Kim M, Kim SY, Noh SM, Song KS, Kim JC and Kim YS: Epigenetic regulation of microRNA-10b and targeting of oncogenic MAPRE1 in gastric cancer. Epigenetics 6 : 740-751, 2011.

37. Stadthagen G, Tehler D, Høyland-Kroghsbo NM, Wen J, Krogh A, Jensen KT, Santoni-Rugiu E, Engelholm LH and Lund AH: Loss of miR-10a activates lpo and collaborates with activated Wnt signaling in inducing intestinal neoplasia in female mice. PLoS Genet 9: e1003913, 2013.

38. Havelange V, Ranganathan P, Geyer S, Nicolet D, Huang X, Yu X, Volinia S, Kornblau SM, Andreeff M, Croce CM, et al: Implications of the miR-10 family in chemotherapy response of NPM1-mutated AML. Blood 123: 2412-2415, 2014.

39. Zhi Y, Xie X, Wang R, Wang B, Gu W, Ling Y, Dong W, Zhi F and Liu Y: Serum level of miR-10-5p as a prognostic biomarker for acute myeloid leukemia. Int J Hematol 102: 296-303, 2015.

40. Kosaka N, Iguchi $\mathrm{H}$ and Ochiya T: Circulating microRNA in body fluid: A new potential biomarker for cancer diagnosis and prognosis. Cancer Sci 101: 2087-2092, 2010.

41. Cortez MA, Bueso-Ramos C, Ferdin J, Lopez-Berestein G, Sood AK and Calin GA: MicroRNAs in body fluids the mix of hormones and biomarkers. Nat Rev Clin Oncol 8: 467-477, 2011.
42. Taylor DD and Gercel-Taylor C: MicroRNA signatures of tumor-derived exosomes as diagnostic biomarkers of ovarian cancer. Gynecol Oncol 110: 13-21, 2008.

43. Cherradi N: microRNAs as potential biomarkers in adrenocortical cancer: Progress and challenges. Front Endocrinol (Lausanne) 6: 195, 2016.

44. Saplacan RM, Mircea PA, Balacescu L and Balacescu O: MicroRNAs as non-invasive screening biomarkers of colorectal cancer. Clujul Med 88: 453-456, 2015.

45. Yu H, Lindsay J, Feng ZP, Frankenberg S, Hu Y, Carone D, Shaw G, Pask AJ, O'Neill R, Papenfuss AT and Renfree MB: Evolution of coding and non-coding genes in HOX clusters of a marsupial. BMC Genomics 13: 251, 2012.

46. Pearson JC, Lemons D and McGinnis W: Modulating Hox gene functions during animal body patterning. Nat Rev Genet 6: 893-904, 2005.

47. Jia H, Zhang Z, Zou D, Wang B, Yan Y, Luo M, Dong L, Yin H, Gong B, Li Z, et al: MicroRNA-10a is down-regulated by DNA methylation and functions as a tumor suppressor in gastric cancer cells. PLoS One 9: e88057, 2014.

48. Ørom UA, Nielsen FC and Lund AH: MicroRNA-10a binds the 5'UTR of ribosomal protein mRNAs and enhances their translation. Mol Cell 30: 460-471, 2008. Attribution 4.0 International (CC BY-NC 4.0) License 